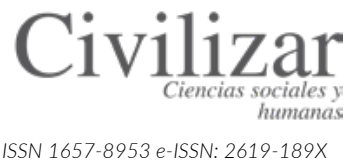

ISSN 1657-8953 e-ISSN: 2619-189X

\title{
La funcionalidad del derecho desde la noción de sistema*
}

\author{
The Functionality of Law from the Notion of System
}

Manuel Antonio Ballesteros Romero

* Artículo de investigación

$\S$ Abogado de la Universidad de Antioquia, Especialista en Derecho Financiero y Negocios de la UPB. Candidato a Doctor en Derecho Universidad de Medellín con tesis de grado titulada "El derecho y el Tiempo". Profesor de Tiempo Completo de la Corporación Universitaria Americana.

Correo electrónico:

mballesteros@coruniamericana.edu.co

(iD) 0000-0001-7176-8912

Cómo citar:

Ballesteros Romero, M. (2020). La Funcionalidad del Derecho desde la Noción de Sistema. Civilizar: Ciencias Sociales y Humanas, 20(38), 95-106. doi: https:// doi.org/10.22518/jour.ccsh/2020.1a07

Recibido: $10 / 01 / 2020$

\section{Resumen}

El punto de partida es la doble opción de abordar el estudio del derecho. De un lado la división mecanicista, que conduce a plantear el derecho como algo puro, al que no se le puede relacionar con las ciencias naturales ni sociales. De otro lado, la visión sistémica, que implica comprender el objeto de estudio en interrelación con el entorno. Se toma partida por la segunda, enfrentando los pensamientos puristas a favor de una visión del objeto de estudio, no como algo que yace en el espacio, sino como algo dinámico, que se manifiesta en el tiempo y se comporta como proceso, que hace parte de ese entorno constituyendo un sistema mayor, y que por la consideración de proceso, elementos del entorno pasan a hacer parte de ese sistema y elementos del sistema pasan a hacer parte del entorno, y que tanto el entorno determina al sistema como el sistema al entorno. El derecho es considerado no como parte del sistema social, sino como una dimensión de dicho sistema y, por ello, inseparable del sistema social; pero, además, tanto el derecho como la sociedad tienen, y requieren para existir, un sustrato donde se encuentran los seres humanos como entidades biológicas, los recursos naturales y el planeta. Por consiguiente, si bien no se puede confundir el sistema social en tanto sistema comunicacional con la agrupación de seres humanos ni con el espacio físico donde existen los seres humanos, no se puede perder de vista que ese sistema físico constituye el sustrato del sistema comunicacional; siendo la cosa en sí, de la cual el sistema social es el fenómeno o el como sí, un mundo construido a partir de la representación del mundo físico.

\section{Palabras clave}

Sistema, mecanicismo, complejidad, sistemas sociales, análisis sistemático, pensamiento sistémico

\section{Abstract}

The starting point is the dual option of approaching the study of law. On the one hand, the mechanistic vision, which leads to posing the law as something pure, which cannot be related to the natural or social sciences. On the other hand, the systemic vision, which implies understanding the object of study in interrelation with the environment. As a starting point, the second option is taken, confronting the purist thoughts in favor of a vision of the object of study, not as something static in space and treated as a thing, but dynamic that manifests itself in time and behave as a process, which is part of that environment constituting a larger system, and that by considering process, elements of the environment become part of that system and elements of the system become part of the environment, and both the environment determines the system and the system the environment. Law is considered a dimensional system and therefore inseparable from the social system; but, in addition, both law and society, have, and require to exist, a substrate where human beings are found as biological entities, natural resources and the planet. Therefore, the social system as a communicational system cannot be confused with the grouping of human beings, or with the physical space where human beings exist, it cannot be forgotten that this real system constitutes the substrate of the communicational system; being the thing itself, of which the social system is the phenomenon or as if.

\section{Keywords}

System, mechanism, complexity, social systems, systematic analysis and systemic thinking 


\section{Introducción}

Este trabajo hace parte de la investigación doctoral sobre el derecho y el tiempo, que pretende, justamente, mostrar el derecho como un algo de la sociedad en que se manifiesta; no en forma estática, sino en forma dinámica, en la misma forma como la sociedad es dinámica al paso del tiempo. Ese algo que es el derecho es entendido, a priori, como un sistema que, a su vez, es constitutivo del sistema social; lo cual entraña una problemática consistente en comprender: a) si el derecho es separable de la sociedad, para estudiarlo como algo puro, o b) si, por el contrario, resulta inseparable y. por lo mismo, no se puede aspirar a encontrar un límite entre derecho y sociedad.

Se podría superar la problemática y cambiarla por una disyuntiva donde ambas premisas pueden ser abrazadas como válidas. La primera desde la visión elementalista o mecanicista fundamentada en el método cartesiano; mientras que la segunda se defendería desde la visión sistémica, según los planteamientos originarios de Bertalanffy. Esa doble posibilidad obliga a tomar partido sobre cómo responde mejor el derecho; no para ser comprendido en sí mismo, sino para comprenderlo desde su funcionalidad en la sociedad, aunque no falte quien quiera defender una posición ecléctica y proponga un sincretismo entre ambas.

Con Kelsen (1945) y su teoría pura del derecho, el mecanicismo alcanzó el paroxismo y a partir de ese momento, pese a que Kelsen ha tenido fuertes y no pocos contradictores, no todos se han distanciado de él en esa visión elementalista. Ese distanciamiento lo demuestra el hecho mismo de que no existan muchas teorías fuertes que planteen un derecho por fuera de la visión cartesiana. Ha habido quienes sostienen que el derecho no es puro, pero muchos lo hacen, contradictoriamente, desde un discurso exclusivamente jurídico, sin darle una oportunidad de ver la interacción entre el derecho y los demás elementos con que coexiste. De hecho, ha sido el mismo Kelsen quien más se ha aventurado a tratar ese tema, en una obra monumental como lo es Sociedad y naturaleza, donde se remonta a las leyes naturales, a los mitos y a las costumbres populares.

Hasta el momento existen importantes estudios del derecho que se apartan de la visión puramente positivista y elementalita, como Zagrebelsky; se destacan también los trabajos de Botero Bernal,
Gutiérrez Prieto y Grün. Es esa línea la que sigue este trabajo: que toma partido por apartarse de la visión mecanicista que hacía análisis sistemático, para abordar un poco de funcionalismo sistémico y tratar de mirar al derecho en la interacción con la química, la física, la biología; no como discursos, ciencias o disciplinas, sino como dimensiones del mundo material donde se desarrolla el ser humano, que es el objeto del derecho. Entendido el derecho no como ciencia, teoría o disciplina, sino como sistema que se manifiesta a través de normas e instituciones.

\section{Metodología}

Se acude a la visión sistémica como un enfoque de abordar los temas objeto de estudio, para lo cual se debe acudir además a un funcionalismo-distópico. El método distópico sirve para preguntarse, por ejemplo: ¿cómo sería la sociedad A en la actualidad si se hubiera suprimido el derecho en un momento dado?, ¿cómo sería un aspecto social A en la actualidad si no hubiera existido una institución jurídica B en el pasado?, ¿cómo sería un aspecto social A en la actualidad si en vez de la institución B que viene existiendo, hubiera existido una institución $\mathrm{C}$ desde el pasado? Esas preguntas distópicas -las cuales son observación práctica de la realidad existente versus la realidad alternativason las que permiten dar aplicación al método funcional desde una pragmática diferente a la propia del método funcionalista.

\section{La visión elementalista}

El hombre ha tratado siempre de conocer su entorno, la parte del mundo que le rodea y con la que interactúa, para valerse mejor de cada elemento del medio. Evocando la cita que Prigogine (2001) hace de Tarnas: "La pasión más profunda del intelecto occidental es encontrar la unidad con las raíces de su propio ser" (p. 12), ilustra que la pregunta por el origen, por la esencia, por el ente, es una preocupación que acompaña el pensamiento occidental desde los presocráticos (Gomperz. 2000. p. 39). Por la pendiente de este interrogante se ha deslizado la razón humana desde los inicios de la filosofía. ¿La cosa en sí es constante, estática, dinámica, mutante, reflejo de un modelo, divisible hasta el infinito, una visión engañosa de nuestros sentidos o nuestra representación?

En este discurrir, lejos de hallar solución, se muestra que al hacer zum de acercamiento a cada interrogante se encuentra que en cada curva 
hay a su vez nuevos meandros, como los encontró Mandelbrot (1996) al responder la pregunta sobre cuánto mide la costa de Bretaña (p. 27). Al respecto de la cual insiste en que:

Es en efecto asombroso -dice- que cuando una bahía o una península que estaban representadas en un mapa a escala 1/100.0oo, se examinan de nuevo en un mapa a $1 / 10.000$, se observa que sus contornos están conformados por innumerables sub-bahías y sub-penínsulas. En un mapa a 1/1000 se ven aparecer sub-sub-bahías y sub-sub-penínsulas y así sucesivamente (p. 34).

Por tanto, esa tendencia a la infinitud que enfrentó Mandelbrot al estudiar un sistema físico se asimila a lo que sucede con cada interrogante: que al abordarlo -tras la aparente resolución a cada interrogante-, se suscitan nuevos problemas, y la solución a cada cuestión viene saturada de nuevas preguntas, y para cada pregunta, se busca un método de resolución (Mandelbrot, 1996, p. 34).

\section{El método elementalista o cartesiano}

El enfoque elementalista pareció por algún tiempo haber dado respuesta a la pregunta sobre cómo llegar al conocimiento de las cosas. El enfoque está fundamentado en el discurso del método de Descartes, especialmente en el segundo y tercer axiomas. El segundo es dividir cada una de las dificultades que examinará, en tantas parcelas como fuere posible y fuere requerido para resolverlas mejor. El tercer axioma se expone como "conducir por orden mis pensamientos, comenzando por los objetos más simples y más fáciles de conocer para subir poco a poco, como por grados, hasta el conocimiento de los más complejos, incluso suponiendo un orden entre aquellos que no se preceden naturalmente los unos a los otros" (Descartes, 1983, p. 6o).

A partir de esos postulados, el pensamiento de la humanidad se inclinó por el estudio de los elementos, como lo resalta Wagensberg (2007) al comentar que "en más de tres siglos de ciencia todo ha cambiado excepto tal vez una cosa: el amor por lo simple. Desde que Galileo, Descartes y Newton inventaran la física, simples han sido los objetos descritos por la ciencia" (p. 11). Ese amor por lo simple encontraba su mejor respuesta en el método cartesiano: llegar a lo más elemental de algo dividiéndolo y fragmentándolo para comprenderlo. Como resultado de ese proceso, señala Botero (2003), la "fragmentación como cosmovisión, inunda tanto los objetos de estudio como los estudiosos de los obje- tos" y agrega: "la fragmentación pasa a ser una cosmovisión generalmente aceptada y particularmente compartida" (p. 44).

Con el cartesianismo nada escaparía de ser fragmentado. En este sentido, "la fragmentación de la realidad implica una concepción del mundo que considera que este puede ser dividido en grupos selectos no solo para ser estudiados sino para estudiar" (Botero, 2003, p. 43) y cada fragmento, a su vez, es subdividido hasta lo más simple. Al respecto, sostiene el mismo autor, "de tal simplicidad -de la ciencia- se deduce buena parte de su prestigio: rigor, universalidad, incluso belleza. El mérito de las ciencias de la naturaleza consistía precisamente en captar la esencia simple e inmutable, pero emboscada tras apariencias superfluas y cambiantes" (Botero, 2003, p. 11).

Así cada subparte se constituyó en objeto de estudio de una nueva disciplina del conocimiento, lo que llevó a las especialidades; y la sociedad exigió especialistas, y no hombres que supieran poco de todas las cosas. Así, en cada área, se prefieren las personas más especializadas, como de manera sentenciosa lo expresa Rifkin (1989): "hemos llegado al extremo en que cada uno de nosotros sabe más y más sobre menos y menos, y, como sociedad, pronto lo sabremos casi todo acerca de casi nada” (p. 118). Esta condición pone, por ejemplo, a un nefrólogo a mucha distancia de un neumólogo y a este, a su vez, distante de un otorrinolaringólogo.

Esa distancia que separa los conocimientos profundos de dos especialistas evidencia que el enfoque elementalista no permite una comprensión holística e integral del sistema. El estudiar las partes y especializarse en cada una de ellas por separado, obviando la existencia de las otras, implica una visión de estas como algo estático, dedicada a una función exclusiva y no interactuante; lo cual resulta en una suplantación del todo por la parte, pues "mientras más partes se especializan en determinado modo, más irremplazables resultan, y la pérdida de la parte puede llegar a la desintegración del sistema total" (Bertalanffy, 1976, p. 72).

Una visión especializada de un problema puede expresar preocupación por el todo, pero siempre estará más preocupado por su segmento concreto de estudio. Eso hará -aun cuando sea muy consciente del medio y del entorno- que, aun sin restarle importancia, un individuo se concentre más en su especialidad, y respecto de esos otros aspectos 
que estén por fuera de su conocimiento específico puede no comprender qué papel cumplen en el todo.

Sobre el paradigma de la especialización se construye la sociedad capitalista, la cual tiene como objetivo maximizar ganancias financieras mediante la optimización de recursos financieros. En este sentido, una empresa debe procurar las mayores utilidades aun destruyendo un poco de naturaleza, reubicando algunas poblaciones, afectando el hábitat de algunas especies y contaminando un poco el agua, siempre que los resultados esperados sean superiores a lo que se proyecta pagar por ese daño, el cual es mirado como una externalidad negativa, que no se quiere causar, pero se causa y se indemniza. Al problema con las comunidades se lleva un especialista - sea trabajador social, sociólogo o antropólogo- según el caso, con una misión específica que debe corresponder con los números de un especialista en administración o finanzas, quien buscan reducir el costo y maximizar la utilidad.

El problema ambiental se aborda de la misma manera, y, en últimas, todos los problemas se reducen a normas jurídicas y precedentes jurisprudenciales, que también son diseñadas especialmente para abordar problemáticas específicas. A partir de eso, la preocupación ya no será el ambiente, sino actuar conforme a la norma ambiental; porque la empresa tiene una comprensión única de la totalidad: la utilidad después de deducir los gastos y los costos -incluidos los costos ambientales y socialesque se cuantifican desde lo jurídico.

El enfoque mecanicista ha enterrado profundamente sus raíces en el derecho, donde se han generado especialidades o áreas -como penal, familia, laboral, administrativo- que, en el caso colombiano, han segmentado no solo la legislación mediante la creación de codificaciones sustantivas diferentes en cada caso, sino incluso creando su propio régimen procesal y jurisdiccional. Así, se encuentra una jurisdicción penal, una jurisdicción de familia, una jurisdicción laboral, una jurisdicción administrativa, una jurisdicción civil y, en cada una, diversos procesos especializados.

Sin embargo, ahí no termina la fragmentación; a la creación de subdivisiones dentro de esas especializaciones, se suma que, de un lado, las áreas a las que no se les asigna una de esas subdivisiones se comportan como áreas huérfanas; como es el caso del derecho ambiental respecto del cual, en un mundo de especialistas, al no existir un proceso específico para lo ambiental ni una jurisdicción ambiental desde lo holístico, las problemáticas ambientales se abordan desde generalidades, limitando el papel de los jueces a lo que dictaminen las corporaciones autónomas regionales; y de otro lado, las subespecializaciones, las cuales, a la vez que facilitan el trabajo del abogado para resolver un problema concreto, hacen difícil que ese mismo profesional tenga una visión holística y prospectiva de la problemática que puede orlar a su cliente al momento de confiarle la administración de sus relaciones jurídicas.

\section{El salto de la visión elementalista a la noción de sistema}

Como es apenas comprensible, la idea no es dar cuenta de la historia del concepto de sistema ni de ser minucioso con la teoría construida. Se trata simplemente de fijar algunas nociones que permitan cumplir el propósito del artículo, de acuerdo con Bertalanffy (1984), padre de la teoría general de sistemas; lo mismo que Luhmann en lo relativo a sistemas sociales.

\section{La visión sistémica}

Con el siglo XX surgen enfoques que controvierten la física clásica y con ello no solo rompen el paradigma de la causalidad, sino que mueven los cimientos que sostenían las ciencias naturales hasta entonces. Para el año 1900 ya se habían desarrollado los planteamientos de la segunda ley de la termodinámica cuando Kepler propone lo que serán las bases de la física cuántica; sin embargo, para 1905 Einstein toma los fundamentos de Kepler para explicar el comportamiento dual del desplazamiento de la luz. Seguidamente, para 1927, Heisenberg expone el principio de incertidumbre y los postulados de la segunda ley de la termodinámica comienzan a usarse para comprender el crecimiento de la entropía en los ecosistemas y en los sistemas sociales.

Aunque se mantiene la forma elementalista de plantearse interrogantes, como el clásico qué es la cosa en sí formulada por Kant, al referirse al ideal de la razón pura (Kant, 1996, p. 260). Paulatinamente, se da un giro epistémico que hace que "la cosa" ya no esté en el corazón del interrogante. Como lo plantea Cereijido (2009), desubicando las cosas del espacio y situándolas en la dimensión temporal: "No somos una cosa sino un conjunto de moléculas que en este momento están presentes en este eterno proceso de ser nosotros” (p. 19) Esta autor enfatiza 
que los sistemas no sufren procesos, sino que son formas adoptadas por los procesos donde la forma es la visión espacial y el proceso es la visión temporal; para ejemplificar esta afirmación, explica que el agua que ingresa al organismo se reparte por los tejidos y luego es expulsada: no es agua que "pasó" por el organismo, sino que ella misma fue el organismo durante el tiempo que participó de los mil y un procesos biológicos (Cereijido, 2009, p. 37).

No existe, entonces, una cosa en sí como oruga, una cosa en sí como crisálida y una cosa en sí como mariposa, sino que además de ese estar en el espacio como cosa, hay un ser en el tiempo como proceso. De esta forma, ubica la cosa - bien sea oruga, crisálida o mariposa - como parte del todo y en función del todo; o bien sea el agua que llena ese cuerpo de la oruga crisálida o mariposa, que no es agua que esté en su cuerpo, sino que hace parte del cuerpo y actúa en función de ese cuerpo que la consume.

Sin esperar reificar el derecho con esos mismos presupuestos, queda preguntarse si el derecho está en la sociedad o es parte de la sociedad; si la libertad, la igualdad o el poder están en la sociedad o son parte de la sociedad.

\section{La teoría general de sistemas}

La teoría general de sistemas surge como una reacción frente a una visión mecanicista-elementalista de las cosas. La teoría consiste, según lo expone su autor, en "el estudio científico de los todos y las totalidades que no mucho tiempo atrás se consideraban nociones metafísicas que trascendían las nociones de la ciencia” (Bertalanffy, 1976, p. 39), quien además precisa que la ciencia de los sistemas concibe un sistema como un conjunto de componentes cuya misión es servir a los propósitos básicos de todo el sistema (Bertalanffy, 1976, p. 315).

Por sistema debe entenderse el conjunto de elementos relacionados entre sí y con el medio ambiente (Bertalanffy, 1976. p. 41). Son sistemas una galaxia, un perro, una célula, un átomo (Bertalanffy, 1984, p. 152). Sin embargo, la relación que debe existir entre las partes debe ser intrínseca -en tanto esa relación le confiere mayor valor o funcionalidad a la parte - a cada una de ellas; esto es, que al interrelacionarse haya, en el todo organizado, algo más que una simple suma de cosas. De la misma manera debe ser la relación entre el todo y el ambiente o entorno.

Esta concepción básica de lo sistémico tenía la virtud de superar el paradigma sistemático imperante desde la antigüedad y que llegara a su paroxismo a partir de Descartes. Dicha superación, dice Piña (2005), ha de entenderse en el sentido de que la concepción sistémica anterior fuese desechada por la nueva, sino lo contrario; pues incorporaba en ella el paradigma anterior, haciendo descansar ahora el concepto en la distinción entre sistema y entorno, pasando de la visión sistemática a la sistémica. Dicho de otro modo, no se negaba que el todo fuera más que las partes, pero se establecía que la distinción capital ya no era todo/parte, sino sistema/entorno (p. 39).

Por esta relación adquiere sentido el apotegma aristotélico, resaltado por Bertalanffy (1976): "el todo es más que la suma de las partes” (p. 58), destacado como una de las características más importantes de los sistemas. No basta que haya un conjunto de cosas y una relación simple entre ellas, sino que se requiere de una relación que haga del todo algo con más energía disponible que el conjunto de todos sus elementos.

Conforme lo anterior, esa interrelación puede ser tan simple o trivial -como la que se observa en un grupo de soldados o en los músicos de una orquesta- o complejas -como puede ser el impacto que genere sustraer o introducir una especie a un ecosistema, sea por ejemplo el caso de los gorriones en la China maoísta o el de los conejos en Australia-. En síntesis, la teoría general de sistemas ve y estudia las cosas no como entes aislados, no como un conjunto separado de elementos, sino, siempre, en relación con su medio, como partes siempre de un todo mayor, de un sistema, y, por lo mismo, en constante interrelación y dependencia con las otras partes.

\section{Clasificación de los sistemas}

Se diferencia entre sistemas materiales y sistemas conceptuales. Los primeros son las entidades percibidas por los sentidos y cuya existencia es independiente del observador. Los segundos son, según Bertalanffy (1979), construcciones simbólicas como la lógica, las matemáticas y la música (p. 47). Según las relaciones con su entorno, los sistemas se clasifican en aislados, los que no intercambian materia ni energía con el medio; cerrados, aquellos que intercambian energía, pero no materia, y abiertos, que según Bertalanffy (1976), tienen como característica el que exhiben importación y exportación, constitución y degradación de sus componentes materiales (pp. 39-146). De este tipo son ejemplos los seres 
vivos, los cuales necesitan entonces de un entorno adecuado con el cual realizar el intercambio de materia.

Para Morín (1992), el entorno para un ser vivo lo conforma un ecosistema, entendido como una comunidad de seres vivos que ocupan un nicho geofísico o medio ambiente. El entorno de un ser vivo está provisto de otros seres vivos, entre los cuales y el medio hay interacciones, tensiones y dependencias, lo cual constituye una autoorganización espontánea a pesar de las aleatoriedades e incertidumbres (Morín, 1992, p. 29).

Todo sistema abierto, por necesitar un entorno, hace parte de un sistema mayor; este sistema conformado por un sistema abierto y su entorno es un sistema cerrado. Luhmann (1998) afirma que a partir de la concepción de límite que separa el sistema del entorno, "ya no se concibe la distinción entre sistemas cerrados y abiertos como oposición sino como relación de gradación" (p. 79); eso hace posible afirmar que todo sistema abierto es potencia de sistema cerrado. Para el humano, su entorno lo constituye la superficie del planeta, este es un sistema cerrado, pues, aunque la luz del sol proporcione la energía necesaria para la vida, el resto de los recursos son limitados; lo cual es la característica propia de los sistemas cerrados, que intercambian energía con el entorno, pero no materia.

En una apretada síntesis y a los efectos que interesan en estas líneas, el pensamiento de Bertalanffy (1976) se funda en dos premisas básicas. La primera es que las cosas existen en realidad con independencia del observador. La segunda es que los sentidos del observador suelen ser traicionados a la hora de captarlas (p. 152). No se trataría aquí de la mera construcción por parte del observador; en cambio, existiría una realidad exterior al observador, que subsiste en sí (Piña, 2005, p. 40). Sin embargo, la constatación de esta realidad no implica que el acceso a ella sea sencillo. Por el contrario, el acceso a dicho conocimiento es imperfecto. Esta falibilidad de la percepción no solo envuelve a las cosas en sí, sino también a su delimitación con el entorno. En este orden, la visión sistémica implica la posibilidad de continuar con el estudio de las cosas, solo que de manera diferente a como lo propone el mecanicismo.

Pero al estudiar las cosas, no desde sus elementos, se renuncia a la simplicidad (elemental) que caracterizaba al elementalismo y, en cambio, queda visible la complejidad del mundo y de los sistemas, donde los múltiples elementos se interrelacionan y lo que eran constantes (ceteris paribus) en el mecanicismo, se convierten en variables que además crecen en forma exponencial.

\section{El enfoque de Luhmann (mirada crítica)}

Parsons es pionero en el estudio de los sistemas sociales y es de una importancia indiscutible. Con Parsons (1966), hablar de la sociedad es hablar de un sistema y hablar de orden social es hablar del orden de un sistema (p. 569). No puede perderse de vista, sin embargo, que este autor es reiterativo en hablar de análisis de los sistemas sociales y efectivamente lo hace; lo que sumado a la jerarquización que hace de los sistemas sociales, deja muy próxima dicha obra a ser un estudio sistemático; es decir, próxima al pensamiento mecanicista.

También Luhmann (1998) se refiere a la jerarquía al interior del sistema. Para él, esa jerarquía no significa remontarse a las instancias ni a una cadena de mando de arriba abajo, significa "que los subsistemas puedan llegar a diferenciar otros subsistemas y que, de esta manera, aparece una relación transitiva del ser contenido en el ser contenido (p. 55).

Hablar de orden social es hablar del orden de un sistema. En esta misma lógica, hablar de una teoría de sistemas en clave luhmanniana es hablar de la teoría de los sistemas sociales. Es decir, "la teoría general de sistemas sociales estará orientada por la teoría general de sistemas; con esto justificamos el empleo del concepto 'sistema'. La teoría de sistemas sociales, por su parte, tendrá pretensiones universales, de aquí que hablemos de "lo general" (Luhmann, 1998, p. 39). Explica Luhmann que la teoría general de sistemas sociales, más que tratar de fijar las características esenciales que se encuentren, sin excepción, en todos los sistemas, más bien formula un lenguaje que introduce problemas y soluciones. Este lenguaje, a su vez, permite comprender que pueden existir distintas respuestas funcionalmente equivalentes a problemas determinados.

La teoría sistémica luhmanniana es funcionalista: aborda el estudio desde la óptica de "sistema y función”. Al respecto, Luhmann (1998) afirma que: "el rendimiento del método funcional y el valor explicativo de sus resultados dependen de cómo se especifica la relación entre el problema y la posible solución. ... Pero el método funcional no consiste simplemente en revelar las leyes de la causalidad" (p. 133). Posteriormente, este autor indica que el 
suyo es "un método comparativo, y su introducción en la realidad sirve para abrir lo existente a otras posibilidades" (Luhmann, 1998, p. 135); luego expresa que: "la experiencia funcional no puede ser por tanto más que el descubrimiento (en general) de la anulación (en concreto) de las equivalencias funcionales" (Luhmann, 1998, p. 136).

Asimismo, Luhmann reitera que: "el concepto de función designa, sin embargo, un estado que va más allá del mero proceso de continuación de la producción autorreferente" (Luhmann, 1998, p. 137), y concluye que: "la teoría de sistemas y la metodología funcional introducen el análisis funcional en primer lugar en la referencia de sistema del sistema de la ciencia” (Luhmann, 1998, p. 234).

El método usado por Luhmann es de análisis funcional, sobre cuya importancia Luhmann es prolijo en todo el Capítulo IV de su obra Sociedad y sistemas (Luhmann, 1998, pp. 130-144). Este tipo de análisis resulta problemático, al menos desde lo semántico, como quiera que la concepción analítica es epistemológicamente opuesta a la comprensión sistémica.

El punto de arranque funcionalista descansa en la presunción de que todo sistema social tiene necesidades de las que pende su subsistencia. Esas necesidades son las únicas explicaciones relevantes de la existencia de todos sus componentes; y todos y cada uno de los elementos que integran un sistema, o los subsistemas que lo conforman, están ahí para satisfacer alguna de esas necesidades. Desde esa funcionalidad es desde donde hay que observarlos. Según Luhmann (1998) "se puede hablar de sistema cuando se tiene ante los ojos características tales que, si se suprimieran, pondrían en cuestión el carácter del objeto de dicho sistema. En ocasiones también se llama sistema al conjunto de dichas características" (p. 29). A ese respecto, asevera Piña (2005) que el funcionalismo, desde la perspectiva de Luhmann no busca acceder a sustancias inmutables, sino al control de alternativas, primero teóricas, luego prácticas. Una vez que se conocen las alternativas -o equivalentes funcionales- que acezan, podemos comenzar a eliminar algunos y a explicar, así, los fenómenos sociales (p. 57).

Para Luhmann (1998), "la teoría general de sistemas no determina las características esenciales que se encuentran sin excepción en todos los sistemas" (p. 46) y plantea que, además de la teoría general de sistemas, pueden construirse otras teorías para un tipo específico de sistemas (Luhmann, 1998, p. 45). Sobre estos planteamientos, este autor se fundamenta para construir una teoría de los sistemas sociales, y afirma: "en este sentido, orientamos la teoría de las ciencias general de sistemas sociales hacia una teoría general de sistemas, y con ello fundamentamos la aplicación del concepto de sistemas" (Luhmann, 1998, p. 47), para la cual se reivindican, a su vez, exigencias de universalidad, razón por la que se califica de general. Esta calificación significaría, en palabras de Luhmann (1998), que cada contacto social tiene que comprenderse como sistema hasta llegar a la sociedad misma como conjunto que toma en cuenta todos los contactos posibles" (p. 47). Además, que ello se podría considerar un absoluto para una teoría pura de la comunicación; por tanto, "fuera del sistema de comunicación de la sociedad no existe comunicación alguna. El sistema es el único que utiliza este tipo de operaciones" (Luhmann, 1998, p. 92). Así, al introducirse la teoría de los sistemas sociales "se excluye la analogía directa entre sistemas sociales, organismos y máquinas" (Luhmann, 1998, p. 45).

Respecto de la clasificación de los sistemas sociales, ella se determina según el proceso de autoselección y fijación de sus fronteras. Dentro de esa clasificación se encuentran: la interacción, que sólo requiere la concurrencia de dos o más sujetos en un mismo lugar y momento; la organización, para la cual el sistema establece ciertos requisitos de conducta que el afiliado se compromete a seguir, y luego el miembro de la organización dirige su comportamiento según las pautas de la organización puesto que ha consentido en ello; y la sociedad, que es el máximo sistema social, integrado únicamente por comunicaciones y por todas las comunicaciones; esta no se conforma sumatoriamente respecto del resto de los sistemas sociales, por el contrario opera en un nivel diferente y superior.

Según Luhmann (1998), la sociedad es el sistema social cuya estructura regula las últimas y fundamentales reducciones con las que el resto de los sistemas sociales pueden conectar. Ella transforma la complejidad indeterminada en complejidad determinada o en complejidad determinable para los otros sistemas y garantiza al resto de los sistemas un entorno domesticado de menor complejidad, un entorno en que la arbitrariedad de lo posible ya ha sido excluida y que plantea, por ello, menores exigencias a las estructuras del sistema (Piña 2005, p. 83). 
Para el sociólogo alemán, el sistema social es un sistema complejo, expresión que le asigna el sentido concreto de sistema adaptativo, como se evidencia en las referencias hechas en el párrafo anterior. En Sociedad y Sistema, Luhmann (1998) escribe: "la distinción conceptual entre sistema y complejidad es crucial para los siguientes análisis precisamente porque se trata de sistemas complejos" (p. 84), y precisa que "los sistemas complejos no solo deben adaptarse a su entorno sino también a su propia complejidad. Debe hacer frente a improbabilidades y deficiencias internas" (Luhmann, 1998, p. 85).

No obstante, el abordaje impecable que este autor hace de la sociedad como sistema complejo deja de lado que el sistema social no solo se adapta al cambio, sino que su adaptación además de ser reactiva, muchas veces es también anticipativa. Es decir, el sistema no solo se adapta al cambio, sino que promueve y produce ese cambio. A ello contribuye fundamentalmente el derecho, que a diferencias de los demás sistemas normativos tiene normas prospectivas que contienen la información sobre la dirección que debe tomar la sociedad.

Además de complejo, el sistema social es autorreferente, entendido como que un organismo que "designa la unidad que presenta para sí misma un elemento, un proceso, un sistema” (p. 87). 'Para sí misma' significa independiente del modo de observación de otros (Luhmann, 1998, p. 89). Afirma Luhmann (1998) que la autorreferencia rige para todo tipo de sistemas, los orgánicos y neurofisiológicos como los psíquicos y sociales constituyentes de sentido: "para todos estos niveles de formación de sistemas rige la ley fundamental de la autorreferencia” (p. 98.)

\section{Entorno y sistema en Luhmann}

Se han señalado las diferenciadoras de la visión de Luhmann, y quedan por destacar la diferenciación enfática que hace entre sistema y entorno. "Hoy en día, en la comunidad científica existe seguramente el consenso de que el punto de partida de cualquier análisis sistémico-teórico tiene que ser la diferencia sistema entorno" (Luhmann, 1998, p. 50). $\mathrm{Al}$ respecto, el autor alemán subordina la autorreferencia a la diferencia entre sistema y entorno: "Sin la diferencia respecto al entorno ni siquiera existiría la autorreferencia, pues la diferencia es premisa para la función de las operaciones autorreferenciales" (Luhmann, 1998, p. 50).
Para Luhmann el entorno no puede ser considerado algo marginal o con un significado asistencial; al contrario, sostiene que la relación con el entorno es constitutiva. Según el autor, desde la visión de la teoría de sistemas autorreferenciales, el entorno es más bien una condición previa de la identidad del sistema, ya que esta es posible únicamente gracias a esa diferencia. (Luhmann, 1998. p. 172). Al respecto, habría que cuestionar que el entorno no puede ser visto únicamente como condición previa de la identidad del sistema, porque el sistema afecta al entorno y en consecuencia también el sistema es una condición de la identidad del entorno. El sistema también es creador del entorno; de no ser así, no se podría hablar de una naturaleza humana como diferente de su naturaleza biológica. Si los concretos seres humanos forman parte no de la sociedad, sino de su entorno, se ven transformados por la sociedad $\mathrm{y}$, a su vez, cambian su entorno tanto material como cultural, hasta el punto de que se podría afirmar que el sistema es una condición previa de la identidad del entorno.

Asimismo, Luhmann (1998) sostiene, que en el marco de la teoría de sistemas temporalizados y autopoiéticos, el entorno es necesario precisamente porque los acontecimientos del sistema cesan en cualquier momento y los acontecimientos posteriores solo pueden producirse con ayuda de la diferencia entre sistema y entorno. Por eso, el autor es conclusivo al afirmar que el punto de partida de todas las investigaciones teóricas que se siguen a partir de esta visión no será una identidad sino una diferencia (Luhmann, 1998, p. 173). En este sentido, Luhmann presenta al sistema como algo que está en el entorno, alimentándose de él sin afectarlo, como en los sistemas cerrados, respecto de los cuales no es relevante el entorno; pero todo sistema abierto es resultado de los microsistemas o subsistemas que los constituyen. En suma, no existe un entorno en sí, independiente de los sistemas, sino que la sumatoria de todos los sistemas son el entorno para cada uno de los sistemas que lo componen, como sistema mayor. Por tanto, sí es importante la diferencia, pero esta será cuestión de ángulos de visión y no una diferencia per se.

El gorrión como sistema, por ejemplo, requiere un entorno. Si se sacan los gorriones en China, se altera ese entorno que es también un sistema y se altera el entorno para las langostas que servían de alimento a los gorriones; el resultado puede ser que altere el entorno para el cultivo de arroz. Un conejo es un sistema abierto, que requiere un entorno, 
cuando se introducen conejos en Australia se altera el entorno que es a su vez un ecosistema.

Como consecuencia de la diferencia sistema-entorno que hace Luhmann (1998), se introduce el concepto de límite. "Aparte de la constitución de elementos propios del sistema, el requisito más importante para la diferenciación entre sistemas sería la determinación de sus límites" (p. 81), los cuales son los que precisamente distinguen el concepto de sistema del concepto de estructura ... cuando los límites están bien definidos, los elementos deben formar parte del sistema o del entorno" (Luhmann, 1998, pp. 78-79). Sin embargo, Luhmann parece prescindir de la dimensión temporal y, por lo mismo, prescindir de la concepción de proceso que experimenta un sistema. La distinción que el autor hace entre sistema y entorno se asemeja a un enfoque analítico, y por lo mismo estático: por ello, plantea que el entorno es condición previa del sistema, pero se minimiza la importancia del contra flujo de información desde el sistema hacia el entorno.

\section{El derecho y la teoría de sistemas de Luhmann}

El concepto de límite que Luhmann mantiene en los sistemas sociales, unido al concepto mismo de sistema social, está integrado solo por comunicación. En este el ser humano no entra, pues se queda constituyendo sistemas orgánicos, ni tampoco la acción humana que va a constituir sistemas psíquicos, los cuales hacen parte del entorno y no del sistema. Debido a estas propiedades, el concepto de límite causa dificultad; de un lado, para comprender que esta visión sea sistémica y no sistemática y, de otro lado, para incluir el derecho como uno de los sistemas a los que se le pueda aplicar la teoría general de los sistemas para ser entendido no como parte, sino como dimensión. El derecho no es la sociedad, pero conforma con esta una totalidad imposible de escindir, de separarlos y establecer los límites entre ambos.

En un momento dado será posible decir qué cosas dentro de la sociedad no son derecho, pero no será posible que esas cosas puedan existir sin el derecho. Tampoco se podrá decir qué cosas dentro del derecho no son sociedad. En palabras de los autores de la teoría sistémico-cibernética del derecho, "el sistema jurídico no es puro, ni mucho menos puede estudiarse como si lo fuera" (Grün y Botero, 2008, p. 54). Por esa misma razón, el derecho no puede estudiarse como una totalidad. Botero (2003, p. 17) sostiene lo equivocado que sería dejar de tratar al derecho como parte de la totalidad para tratarlo como la totalidad misma, y por eso no tiene límites. Retomando a Botero y Grün (2008), se dirá que un sistema de segundo orden, una modelación no podría creer ni proponer modelos de lectura "como si” el sistema de primer orden estudiado (eld) fuera puro. Entonces, ni en el primer grado ni en uno segundo, el derecho es puro (Grün y Botero, 2008, p. 56). Una visión del derecho como algo puro es una visión estática del derecho, una visión en condiciones ceteris paribus, que sitúa al derecho en el mundo de las formas, pero lo aleja del mundo de los procesos. Al respecto, Einstein (1993), al confrontar los argumentos de Newton, exponía que:

El universo no nos ha sido dado dos veces, con una tierra en reposo y con una tierra en movimiento, sino una sola vez, con sus movimientos relativos, que es lo único susceptible de ser determinado. En consecuencia, no es lícito aventurar juicios acerca de cómo serían las cosas de no estar animada la tierra de un movimiento de rotación (p. 31)

Haciendo analogía con Einstein, se podría afirmar que no nos han sido dados dos derechos, uno para la sociedad y otro para estudiarlo. La entidad del derecho impide separarlo de la sociedad sin que se desvanezca en ese mismo acto. ¿Se podría hablar de sociedad sin derecho?, ¿cómo sería? Una determinada sociedad se puede delimitar y dentro de ella se pueden delimitar otros sistemas: estratos, etnias, edades, géneros; quizá los hospitales, quizá las cárceles; se puede separar la población y el sistema vial; pero el derecho -como sistema comunicacional de primer orden- nunca se podrá separar de la sociedad. No se puede establecer un límite; es decir, hasta aquí llega el derecho sin sociedad y a partir de aquí es sociedad sin derecho.

Si se hiciera un ejercicio de separar sociedad y derecho, ambos dejarían de existir. El derecho, al no ser una totalidad, no tiene existencia en sí mismo, por fuera de la sociedad; pero tampoco es una parte de la sociedad que se pueda separar de ella, como se puede separar un órgano del cuerpo, o la población del territorio, o como se puede separar el oxígeno del hidrógeno en la molécula de agua. En cambio, no hay sociedad si no hay derecho y no hay derecho si no hay sociedad, sin que sean la misma cosa derecho y sociedad con condición de existencia uno del otro.

El derecho es una dimensión -espacio temporal- de la sociedad. Como la profundidad, la anchura o la altura son a los cuerpos, el derecho es 
a la sociedad. Sin la dimensión jurídica no hay sociedad y sin esta no hay derecho. No puede verse tampoco a la sociedad como una mezcla de la cual uno de sus componentes sea el derecho, porque la mezcla tiene como característica que los elementos que la constituyen existen previamente por separado; pero no sucede eso con la sociedad y el derecho.

Muy ilustrativo para entender el crecimiento simultáneo e indisoluble del derecho como dimensión de la sociedad resulta un prolífico diálogo entre Ricoeur y Changeux (2001), titulado La naturaleza y la norma. Igual que con el derecho, sucedería con el poder. Tal como afirma Foucault (1994): "no sabemos quién lo tiene exactamente -el poder-, pero sabemos quién no lo tiene" (p. 15), y un tanto igual sucedería con la libertad.

Por tanto, cómo establecer un límite al poder o a la libertad, de manera que podamos decir: esto y solo esto es poder, y aquello y solo aquello es libertad, siendo que ambos constituyen la sociedad, pero también constituyen el derecho. ¿Cómo sería una sociedad en ausencia del poder? ¿Cómo sería una sociedad en ausencia de libertad? ¿Cómo es una sociedad en ausencia del derecho? Estas preguntas llevan implícitas una afirmación indefinida de lugar y de tiempo del siguiente sentido: iNunca $y$ en ningún lugar es pensable una sociedad sin derecho!

Si el método de Luhmann no es el analítico, sino funcionalista, habría que preguntarse qué función cumple el derecho. Esto es, ¿para qué o para quienes son las comunicaciones que establece? ¿Para quién garantiza el cumplimiento de expectativas normalizadas? ¿A quién le prohíbe conductas cuando las prohíbe? ¿Qué significa que el derecho sea válido o qué esté acompañado del símbolo de validez? ¿Para qué el derecho institucionaliza los tribunales? Sería ambiguo suponer que el derecho esté en función del ser humano como sistema psíquico o como sistema orgánico, porque ese humano -en la visión de Luhmann - no es sistema sino entorno: las entidades corpóreas, perceptibles por los sentidos, sobre los cuales se satisface la entidad conceptual derecho subjetivo, el cual también está en el entorno. Esta consideración llevaría a que el sistema estuviera en función del entorno.

Si el humano es sujeto de derecho, ni como sistema orgánico ni como sistema psíquico, este puede estar por fuera del límite del sistema social -aunque se exprese que el humano no es comunicación ni es información-. Eso implica que, aun cuando solo la comunicación comunique y solo en la sociedad haya comunicación, no será solo la comunicación lo que constituya la sociedad; ni será la comunicación quien realice, per se, el acto de comunicar, aunque la realice para sí misma. El humano, ni como sujeto ni como ciudadano ni como persona, es entidad biológica, orgánica o psíquica. Cada una de esas categorías es una construcción cultural, concretamente construcción de ese sistema cultural que es el derecho. Incluso el esclavo, cuando es considerado un bien - no sujeto sino objeto- es una construcción jurídica y, por lo mismo, hace parte de ese sistema comunicacional.

La cultura crea espacios y tiempos abstractos a partir del espacio-tiempo termodinámico; ese mundo abstracto es un mundo normalizado. Se normaliza la gastronomía, la caza, la pesca, la vivienda, la incidencia de las estaciones en los cultivos, de los periodos lunares en las cosechas, la ubicación de las estrellas para la navegación. Se normaliza el apareamiento, incluso la reproducción de las especies domesticadas. Se normaliza el tiempo, el espacio, el peso, el volumen, creando patrones: días, metros cúbicos, kilogramos, etcétera. Se sistematizan los procesos para normalizar los inventos y descubrimientos, aun cuando a los mismos se llegue por serendipia. Sobre ese mundo abstracto normalizado, se recrea, por el derecho, un mundo jurídico; sobre el espacio cultural, se crea el espacio jurídico; sobre el tiempo cultural, se crea el tiempo jurídico; sobre las entidades culturales, se crean los bienes jurídicos; de la familia biológica, se reinventa la familia cultural y de esta se reinventa la familia jurídica; y sobre el humano cultural, se crea al sujeto de derecho.

La sociedad comunica y la norma informa. Toda norma es información, y normalizar es la forma más eficiente de informar. La cultura no se genera cuando el humano introduce o aprovecha los cambios que sufre la naturaleza, sino cuando se apropia de eso conocimiento y puede transmitir cómo transforma, adapta, interpreta y representa ese mundo físico. La importancia de transmitir ese conocimiento no es espacial; es decir, de un pueblo a otro; es temporal, de una generación a otra. En el espacio, hay transmisión de tecnología, lo cual no requiere un lenguaje elaborado; pero entre las diferentes generaciones de un mismo pueblo no se transmiten ni se heredan herramientas, se transmiten y heredan conocimientos, y eso requiere convertir ese conocimiento en información, lo que exige 
códigos de comunicación mucho más elaborados y normalizar todos los conocimientos que se quieran transmitir.

La información o norma jurídica se caracteriza porque no es útil en el plano individual, sino en el intersubjetivo; sin embargo, a diferencia de cualquier otra norma que transmita información intersubjetiva, esta información se refiere a la titularidad de intereses individuales que pueden ser disputados por el otro. Cuando dos personas cocinan, dialogan, juegan o compiten, sin poner en riesgo sus bienes, esas relaciones pueden estar informadas por normas sobre el lenguaje, la recreación, el deporte, pero no por norma jurídica. El derecho construye el sujeto al construir la titularidad, la cual no está en el mundo físico, y la norma informa sobre esa titularidad, a partir de la categoría de derechos subjetivos. Estos derechos solo pueden ser comprendidos por el entendimiento, pero recaen sobre un sustrato corpóreo, que es el bien, el cual puede ser percibido por los sentidos, aunque sea inmaterial, pues puede ser una canción, un olor, un color, la vida, la libertad. La titularidad crea sujetos - que son comunicación- y crea objetos -que también son comunicación- y la norma informa sobre esa relación. De manera que ni el sujeto ni el objeto de derecho están en el entorno, sino en el sistema.

\section{Conclusiones}

A lo largo de estas reflexiones se ha tratado de hacer una diferenciación entre la visión elementalista que caracterizó todo estudio durante la modernidad y la visión sistémica que ha ganado un importante reconocimiento a partir de la segunda mitad del siglo XX y en la cual se enmarca este trabajo. Resulta valioso, al abordar cualquier objeto de estudio, saber y diferenciar si se usa un enfoque elementalista (mecanicista) o si, por el contrario, se usa un enfoque sistémico, entendiendo, entre otras cosas que:

- Aunque tanto el mecanicismo como la visión sistémica se refieren a su objeto de estudio como sistema, el primero aborda ese objeto de manera sistemática, analítica o fragmentada, mientras que la visión sistémica es holística e integradora.

- Una comprensión sistémica riñe con un abordaje analítico del objeto de estudio, pero también con una teoría pura de los sistemas y, más, con una teoría pura especializada en sistemas sociales, sin que eso impida ver los rasgos diferenciadores entre sistemas y sobre todo entre los sistemas físicos y los conceptuales y al interior de estos, entre los sistemas comunicacionales; como es el caso de los sistemas sociales o, incluso, entre sistema y entorno.

- Una comprensión sistémica riñe con abordar su objeto de estudio de manera exclusiva como una "cosa" en un plano puramente espacial y, por ende, estática, y privilegia abordar su objeto de estudio como "proceso" en una dimensión temporal $\mathrm{y}$, por tanto, dinámica.

- El enfoque sistémico riñe con la visión exclusivamente causalista, propia del mecanicismo y comprende que el sistema es complejo, entendido no en el sentido de pensamiento complejo, sino de ciencia de la complejidad.

- El enfoque sistémico del objeto de estudio resulta más difícil porque implica abordar la complejidad, pero tiene como retribución la comprensión holística que prevé, desde el principio, las externalidades.

- Es preciso marcar una diferencia importante entre una comprensión teórica de los sistemas, como puede ser la comprensión luhmanniana de límites del sistema que no se corresponden con la comprensión praxeológica de los sistemas abstractos; por ejemplo, una comprensión del derecho como dimensión social no admite la idea de límites.

- Una comprensión sistémica del derecho, abordado como dimensión social, resulta explicar mejor la relación derecho-sociedad que viene a ser fundamental en esta investigación.

- Se preferirá la visión sistémica del derecho, que obliga a estudiarlo integrado con todo acontecimiento. De manera que impide aislar el derecho y pretender observarlo como algo puro y que exige comprenderlo como una dimensión -espacio temporal- de la sociedad y por lo mismo, sin límites con el sistema social y sin límites frente al sustrato material sobre el que recaen las comunicaciones de la sociedad. Por ejemplo, el homicidio recae en una entidad percibida por los sentidos que es la vida y esa vida, a diferencia de la vida de otros seres, interesa al derecho porque tenía una titularidad jurídica; las normas de sismo 
resistencia a las que se refiere el derecho tienen sentido solo en cuanto están vinculadas al comportamiento geológico del planeta y puedan afectar la titularidad de un sujeto.

- Si bien no se puede confundir el sistema social, como sistema comunicacional, con la agrupación de seres humanos ni con el espacio físico donde existen los seres humanos, haciendo parte de un sistema material, no se puede perder de vista que ese sistema material constituye el sustrato del sistema comunicacional, siendo la cosa en sí de la cual el sistema social es el fenómeno o el como sí.

- El mundo jurídico si bien reinventa espacio y tiempo, tiene sus cimientos en el mundo cultural y este, a su vez, en el mundo termodinámico, donde yacen seres vivos sin titularidad, excluidos de la categoría de sujeto, y sin que sobre ellos recaiga titularidad. Ese mundo carece de protección jurídica y reclama titularidad. Ese mundo es aproximado de manera acelerada al ápex entrópico, y la sociedad lo mira indiferente porque solo lo considera el entorno del entorno del entorno.

\section{Referencias}

Bertalanffy, L. (1976) Teoría general de los sistemas. Fondo de Cultura Económica.

Bertalanffy, L. (1979). Perspectivas en la teoría general de sistemas. Alianza editorial.

Bertalanffy, L. (1984). Tendencias en la teoría general de sistemas. Alianza Editorial

Botero, A. (2003a). Nuevos paradigmas científicos y su incidencia en la investigación jurídica. Diálogos de saberes No. 18-19. Centro de Investigaciones Socio Jurídicas, Facultad de derecho, Universidad Libre (Bogotá). http://www.dirittoequestionipubbliche. org/D_Q-4/studi/studi_Botero-Bernal.pdf

Botero, A. (2003b). Diagnóstico de la eficacia simbólica del derecho en Colombia y otros ensayos. Señal Editora.

Cereijido, M. (2009). Elogio del desequilibrio. Siglo XXI editores

Descartes, R. (1983). Discurso del método. (A. Rodríguez Huéscar, Trad.). Orbis editores.

Einstein, A. (1993). La teoría de la relatividad. Altaya.

Ferrater, J. (2004). Diccionario de filosofía. Tomo I. Editorial Ariel

Foucault, M. (1994). Un diálogo sobre el poder y otras conversaciones. Altaya.
García J. (1989). Entropía/Lenguajes. Buenos Aires: Ed. Hachette.

Gomperz, T. (2000). Pensadores griegos. Tomo I. Editorial Herder.

Grün, E. y Botero, A. (2008) Hacia una teoría sistémico-cibernética del derecho. Universitas, 117. 41-64. https://repository.udem.edu.co/handle/11407/328o?show=full

Guarner, F. (2007). Nutrición Hospitalaria. Memorias de historia antigua VII. 22.

http://scielo.isciii.es/scielo.php?script=sci_arttext\&pi$\mathrm{d}=$ So212-16112007000500003

Kant, E. (1996). Crítica de la razón pura. Editorial Porrúa.

Kelsen, H. (1934). Teoría general del Estado. (L. Legaz Lacambra, trad). Editorial Labor.

Kelsen, H. (1945). Derecho y sociedad. Una investigación sociológica. (J. Perriaux, trad.). Editorial Depalma.

Latorre, E. (1996). Teoría General de Sistemas. Cali: Ed. Universidad del Valle.

Luhmann, N. (1998). Sistemas sociales. Lineamientos para una teoría general. Editorial Anthropos.

Mandelbrot, B. (1996). Los objetos fractales. Tusquets Editores S.A.

Mandelbrot, B., y Hudson, L. (2006). Fractales y Finanzas. Trad. Ambrosio García Leal. Barcelona: Tusquets Editores S.A.

Morín, E. (1992). El Paradigma de lo perdido. Editorial Kairós.

Morín, E. (1996). Introducción al pensamiento complejo. Barcelona: Gedisa editorial.

Parsons, T. (1966). Sistema social. Editorial Revista de Occidente.

Piña, J. I. (2005). Rol social y sistema de imputación. Una aproximación sociológica a la función del derecho penal. Universidad de Navarra.

Prigogine, I. (2008). Las leyes del caos. (J. Vivanco, trad.). Ed. Crítica.

Ricoeur, P. y Changeux, J. (2001). La naturaleza y la norma: lo que nos hace pensar. Fondo de Cultura Económica.

Rifkin, J. (1990). Entropía. Editorial Urano.

Schopenhauer, A. (1995). Sobre la Voluntad de la Naturaleza. Grandes Obras del pensamiento, No. 65. Barcelona: Ed. Altaya.

Wagensberg, J. (2007). Ideas sobre la complejidad del mundo. Tusquets Editores S.A 